\title{
Two hyperparasitaemic Plasmodium falciparum cases successfully treated with Artemisinin-based Combination Therapy
}

\section{Benudhar Mukhi}

ICMR-National Institute of Malaria Research, New Delhi, India

\section{Anupkumar R. Anvikar}

ICMR-National Institute of Malaria Research, New Delhi, India

\section{Bina Srivastava}

ICMR-National Institute of Malaria Research, New Delhi, India

\section{Himanshu Gupta}

Department of Infection Biology, London School of Hygiene and Tropical Medicine, Keppel Street, London, United Kingdom

Susanta Kumar Ghosh ( $\nabla$ ghoshnimr@gmail.com )

ICMR -National Institute of Malaria Research, Bangalore Field Unit, India

\section{Case Report}

Keywords: Plasmodium falciparum, severe malaria, hyperparasitaemia, hypergametocytaemia, artemisinin-based combination therapy, primaquine

Posted Date: October 20th, 2020

DOl: https://doi.org/10.21203/rs.3.rs-95230/v1

License: (9) This work is licensed under a Creative Commons Attribution 4.0 International License. Read Full License 
Title

Two hyperparasitaemic Plasmodium falciparum cases successfully treated with Artemisininbased Combination Therapy

\section{Authors}

Benudhar Mukhi ${ }^{1 \#}$, Anupkumar R. Anvikar ${ }^{1}$, Bina Srivastava ${ }^{1}$ Himanshu Gupta $^{2}$ Susanta Kumar $\mathrm{Ghosh}^{3 \# *}$

\section{Authors' Affiliations}

${ }^{1}$ ICMR-National Institute of Malaria Research, New Delhi, India.

${ }^{2}$ Department of Infection Biology, London School of Hygiene and Tropical Medicine, Keppel Street, London, United Kingdom.

${ }^{3}$ ICMR -National Institute of Malaria Research, Bangalore Field Unit, India.

ICMR- Indian Council of Medical Research

\# Manipal Academy of Higher Education, Manipal - 576104, Karnataka, India

\section{*Correspondence}

Susanta Kumar Ghosh, (E-mail:ghoshnimr@gmail.com)

\section{Email addresses of all authors}

1. Benudhar Mukhi: benudhar.mukhi@gmail.com

2. Anupkumar R. Anvikar: anvikar@gmail.com

3. Bina Srivastava: shbira@gmail.com

4. Himanshu Gupta: Himanshu.Gupta@1shtm.ac.uk

5. Susanta Kumar Ghosh: ghoshnimr@gmail.com 


\section{Abstract}

\section{Background}

Hyperparasitaemia is an important event in a cascade of Plasmodium falciparum severe malaria (SM) but requires host responses to cause cerebral malaria (CM) leading to death, if left untreated. Here, we report two hyperparasitaemic patients with no CM.

\section{Methods}

Malaria diagnosis was performed based on thick and thin smears examination, and immunochromatographic-based rapid diagnostic test assay. Parasitaemia was calculated following World Health Organization (WHO) protocol. Haematological and biochemical investigations were also performed.

\section{Results}

The first patient had $42 \%$ parasitaemia (100\% asexual parasites). The second one had $9.5 \%$ parasitaemia comprising $46 \%$ asexual, and 54\% sexual stages with 1:1 male to female ratio. On the day of admission, both had presented abnormal haematological and biochemical parameters compared to the reference ranges. Remarkably, both the patients recovered successfully with oral artemisinin-based combination therapy (ACT) and a single dose of primaquine.

\section{Conclusion}

The presence of hypergametocytaemia may hinder the elimination efforts, if not treated immediately. This report also signifies the need of accurately estimating the parasitaemia in malaria patients and their timely management to prevent complications and mortality.

Keywords: Plasmodium falciparum, severe malaria, hyperparasitaemia, hypergametocytaemia, artemisinin-based combination therapy, primaquine 


\section{Background}

In Plasmodium falciparum infection, $>10 \%$ parasitized red blood cells (RBCs) generally known as hyperparasitaemia, one of the features leading to severe malaria (SM) [1]. Cerebral malaria (CM), severe anemia, multiple organ dysfunctions, and respiratory failure are the common complications with severe $P$. falciparum infection, suggestive of sequestered parasites in the vital host organs, and a threat for recrudescent infections [2,3]. Hyperparasitaemia has also been associated with high gametocyte carriage, [4] and de novo anti-malarial drug resistance, [5] which have implications on malaria elimination programme. Generally, hyperparasitaemia is found in non-immune, [6] and semi-immune individuals [7]. In Thai population, hyperparasitaemia was associated with poor prognosis in adults suffering from CM [8] whereas, in Nigerian children, it increased the risk of CM [9]. In Indian patients, hyperparasitaemia has been correlated with amplified risk of mortality with multi-organ dysfunction (MOD) [10], and patient's death within a short period of time [11]. Here, we report two Indian adult patients, who had P. falciparum hyperparasitaemia with no severe or adverse events - one of them presented hypergametocytaemia stages.

\section{Methods}

\section{Study design}

The two prospective adult males infected with hyperparasitaemia of $P$. falciparum visited the malaria clinic at the Wenlock District Government Hospital, Mangalore, Karnataka, India for diagnosis and treatment. Clinical, biochemical, hematological, parasitological outcomes as well as treatment details of the two patients are presented in this brief report. Indian reference value system for these parameters was considered [12].

\section{Malaria diagnosis and quantification of parasites}

Both thick and thin blood smears on glass micro slides were prepared from spirit-swabbed finger pricking [13]. These smears were stained with 10\% Giemsa and examined under 100X immersion oil objective lens using Carl Zeiss Primo Star light microscope (Germany) [14]. Two trained expert microscopists examined both thick and thin smears for diagnosis and determination of parasite counts the senior expert (SKG) reconfirmed the final results. The mean values of two counts were taken for estimating final parasitaemia. The percent parasite density in the thin films was determined by counting the number of infected RBCs examined against 5000 RBCs in 20 microscopic fields considering the actual number of $\mathrm{RBCs} / \mu \mathrm{L}$ for each patient i.e. 3.87 and $3.69 \mathrm{million} / \mathrm{mm}^{3}$, respectively. Similarly, parasitaemia per $\mu \mathrm{l}$ of blood in the thick smears was calculated enumerating the number of parasites counting 200 white blood cells (WBCs) in relation to the actual number of WBCs/ $\mu$ l i.e. 6900 and $6000 / \mathrm{mm}^{3}$, respectively. 


\section{Laboratory procedures}

On admission, $4 \mathrm{ml}$ venous blood (pre-treatment) from the two patients was collected in EDTA vacutainers (BD Vacutainer®; Cat. No. 367861) for haematological tests. Another 4ml blood was collected in Clot Activator Vacutainer (BD Vacutainer ${ }^{\circledR}$ 367812) for biochemical analyses for liver and kidney function tests. DxH 800 Hematology (Beckman Coulter) and Cobas® 6000 (Roche) analyzers were used for hematological and biochemical tests, respectively. All the tests were performed at the National Accreditation Board for Testing and Calibration Laboratory (NABL)-accredited laboratory services at Kasturba Medical College, Mangalore, India.

\section{Treatment}

Both the inpatients were treated orally with artemisinin-based combination therapy (ACT) - artesunate $200 \mathrm{mg}$ and $1500 \mathrm{mg}$ sulfadoxine plus $75 \mathrm{mg}$ pyrimethamine on day 0; artesunate $200 \mathrm{mg}$ on day 1 and day 2, respectively, followed by a single dose of $45 \mathrm{mg}$ primaquine on day 2 as per the guideline of national programme [15]. All required safety measures and prognosis of oral ACT therapy were monitored in the hospital as per the protocol.

Both the patients were discharged from the hospital on day 7 when blood smears were found negative or other parameters showed normal.

\section{Results}

\section{Clinical manifestations}

The two adult patients aged 35 and 36 years, respectively presented almost similar clinical manifestations having high-graded fever of $39^{\circ} \mathrm{C}$ and $38.5^{\circ} \mathrm{C}$, respectively. On admission both the patients had higher heart rates and abnormal breathing issues. Clinical assessment revealed no neurological and lymphatic abnormalities, but had palpable splenomegaly on admission. Detailed information on physical and clinical manifestations is presented in Table 1. 
Table 1. Physical and clinical characteristics of two adult patients

\begin{tabular}{lcccccc}
\hline Parameter & \multicolumn{2}{c}{ Patient 1 } & \multicolumn{3}{c}{ Patient 2 } \\
\hline & Day 0 & Day 1 & Day 7 & Day 0 & Day 1 & Day 7 \\
\cline { 2 - 7 } Weight (kg) & 66 & 65 & 64.5 & 60 & 60.8 & 59 \\
Height $(\mathrm{cm})$ & 165 & 165 & 165 & 162 & 162 & 162 \\
Blood Pressure (mmHg) & $130 / 70$ & $150 / 100$ & $124 / 84$ & $140 / 90$ & $130 / 88$ & $126 / 82$ \\
Axillary temperature & $39^{\circ} \mathrm{C}$ & $37.5^{\circ} \mathrm{C}$ & $36.8^{\circ} \mathrm{C}$ & $38.5^{\circ} \mathrm{C}$ & $37.2^{\circ} \mathrm{C}$ & $36.4^{\circ} \mathrm{C}$ \\
Heart rate (bpm) & 100 & 84 & 72 & 88 & 84 & 76 \\
Eyes & Burning sensation & Normal & Normal & Burning sensation & Normal & Normal \\
Throat & Sore & Normal & Normal & Sore & Normal & Normal \\
Chest & Burning sensation & Normal & Normal & Burning sensation & Normal & Normal \\
Neurological system & Normal & Normal & Normal & Normal & Normal & Normal \\
Lymphatic system & Normal & Normal & Normal & Normal & Normal & Normal \\
Chills & Yes & Yes & No & Yes & Yes & No \\
Headache & Yes & Yes & No & Yes & Yes & No \\
Nausea & Yes & No & No & Yes & No & No \\
Vomiting & Yes $(3$ times) & No & No & Yes (>3 times) & Yes & No \\
Cough & Yes & Yes & No & Yes & No & No \\
Shivering & Yes & No & No & Yes & No & No \\
Loss of Appetite/ & Yes & Yes & No & Yes & No & No \\
Anorexia & & & & & & \\
Fatigue & Yes & Yes & Yes & Yes & No & No \\
Myalgia (back and & No & No & No & No & No & No \\
limbs) & & & & & & \\
Jaundice & Yes & Yes & No & Yes & No & No \\
Hepatomegaly & No & No & No & No & No & No \\
Splenomegaly & Palpable & Palpable & No & Palpable & Palpable & No \\
\hline & & & & & & \\
& & & & & \\
& & & & & &
\end{tabular}

\section{Haematological and biochemical analyses}

On the day of admission i.e. day 0 both the patients presented abnormal haematological and biochemical parameters compared to the reference range (Table 2). However, total leukocyte count, total protein, albumin and globulin and their ratios were within the reference ranges. Patient 2 had severe anemia and acute kidney injury. Both patients presented hypoglycemia and clinical jaundice. Further details on haematological and biochemical test results are shown in Table 2. 
Table 2. Serological, haematological, and biochemical test results of two adult patients.

\begin{tabular}{|c|c|c|}
\hline Parameter & Patient 1 & Patient 2 \\
\hline HIV & Negative & Negative \\
\hline HBsAg & Negative & Negative \\
\hline $\mathrm{Hb}(\mathrm{g} / \mathrm{dl})$ & 11.1 & 6 \\
\hline TLC $\left(\right.$ per $\left.\mathrm{mm}^{3}\right)$ & 6900 & 6000 \\
\hline PCV \% & 34.9 & 30 \\
\hline Total RBC (million $/ \mathrm{mm}^{3}$ ) & 3.87 & 3.69 \\
\hline Platelet counts (per $\mathrm{mm}^{3}$ ) & 24000 & 50000 \\
\hline Blood sugar (mg/dl) & 45 & 49 \\
\hline Serum urea $(\mathrm{mg} / \mathrm{dl})$ & 58.4 & 49 \\
\hline Serum creatinine $(\mathrm{mg} / \mathrm{dl})$ & 0.66 & 11 \\
\hline Total bilirubin (mg/dl) & 8.37 & 3.89 \\
\hline Direct bilirubin (mg/dl) & 3.5 & 2 \\
\hline Indirect Bilirubin (mg/dl) & 4.87 & 1.95 \\
\hline AST levels (IU/L) & 96.5 & 96 \\
\hline ALT levels (IU/L) & 70 & 56.7 \\
\hline Alkaline Phosphate (IU/L) & 563.3 & 389.9 \\
\hline Total Protein (g/dl) & 6.54 & 7.91 \\
\hline Albumin (g/dl) & 3.85 & 3.98 \\
\hline Globulin (g/dl) & 2.69 & 2.5 \\
\hline A:G Ratio & 1.43 & 2 \\
\hline Parasitaemia/ $\mu \mathrm{l}$ & $16,68,824$ & $3,04,000$ \\
\hline Asexual \% & $100 \%$ & $13,6,800(46 \%)$ \\
\hline Sexual \% & 0 & $16,7,200(54 \%)$ \\
\hline Male gametocyte $\%$ & 0 & $51 \%$ \\
\hline Female gametocyte $\%$ & 0 & $49 \%$ \\
\hline
\end{tabular}




\section{Patient 1}

The microscopic examination of peripheral blood smears confirmed only asexual stages of $P$. falciparum parasites based on typical morphological characteristics. Parasitaemia was $1668824 / \mu 1$ of blood based on thick smear examination and $42 \%$ on thin smear. Fig. 1 A and B show thick smear images, and Fig. $1 \mathrm{C}$ and D are thin smear images. A large number of typical chromatin dots of ring form are seen on thick smears (Fig. $1 \mathrm{~A}$ and B). The parasitological assessment of thin smear for species identification was the cytoplasm, which makes the complete ring formation in young trophozoites followed by; thickening and invariably contains several vacuoles to develop the trophozoites. The chromatin (parasite nucleus) was characteristically contained as a single bead, double bead forms on thin smear examination (Fig. $1 \mathrm{C}$ ). The most important feature of the rings was found on the margin or edge of the red blood cells, called 'accolé/appliqué' forms (Fig 1. C and D). Accolé forms were seen in early stage of $P$. falciparum parasites and these are three distinct types - common form, rim form and displaced form (16\%). The large mass of golden brown pigment (haemozoin) was seen in the pre-schizont and schizont stage (20\%). A low number of pigmented monocytes $(0.19 \%)$, neutrophils $(0.29 \%)$, and eosinophils $(0.09 \%)$ was detected (Fig. 1 A). The unusual of marked multi parasitism (74.02\%, 1 parasite/RBC; 14.95\%, 2 parasites/RBC; 7.7\%, 3 parasites/RBC; 3.14\%, 4 or more parasites/RBC; 0.19\%) was observed (Fig. $1 \mathrm{C}$ and D). No sexual forms i.e. gametocytes were detected in this patient. 

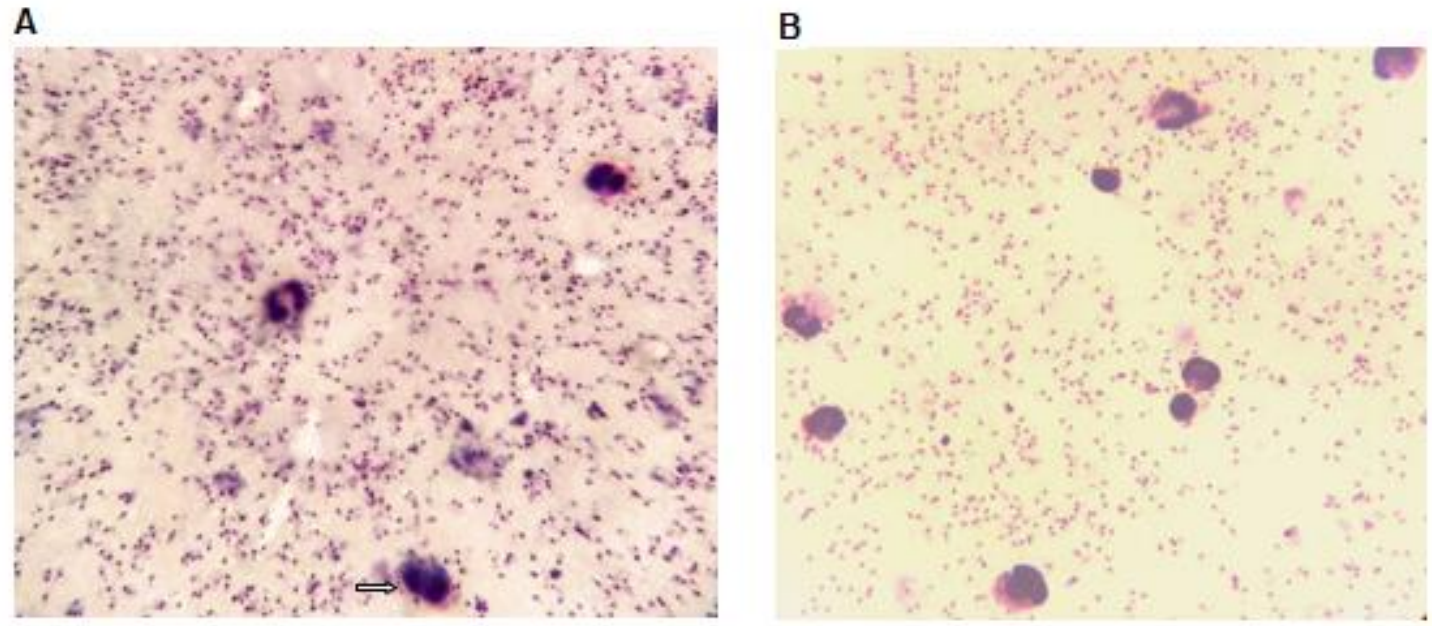

C

D
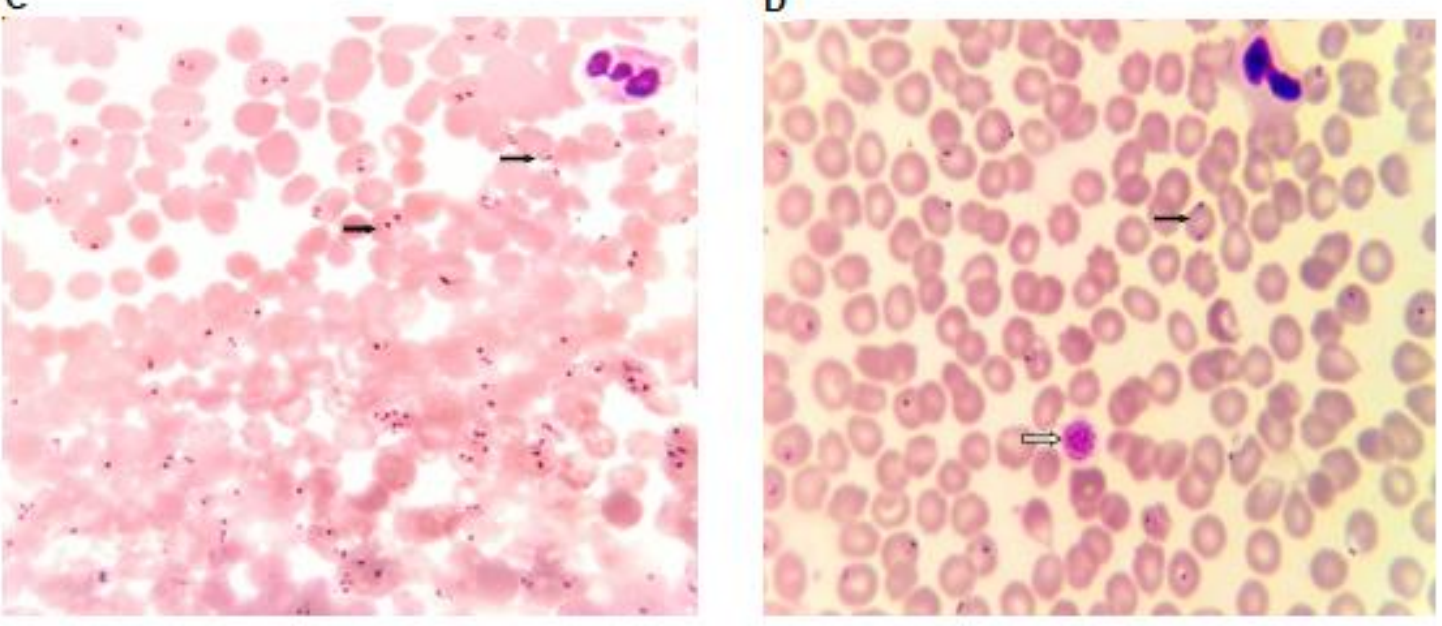

Fig. 1: Images of asexual form of $P$. falciparum parasites $X 1000$ (Patient 1)

Panels A and B show thick smear images with huge ring form of $P$. falciparum parasites. Panels $\mathrm{C}$ and $\mathrm{D}$ on thin smears. Accolé form is seen in both $\mathrm{C}$ and $\mathrm{D}$. A double-chromatin ring is seen in panel $\mathrm{C}$ (arrow in the middle). X 1000

\section{Patient 2}

Patient 2 had mixed stages of parasites. Total parasitaemia on thick smear was 304000/ $\mu \mathrm{l}$ of blood, and 9.5\% on thin smears (Fig. $2 \mathrm{~A}$ to D). Infected RBCs in thin smear were normal in size and contained young rings, and in some mature stages, showing occasionally thin accolé/appliqué forms (Fig. 2 C). Asexual stages constituted about $46 \%$ of $9.5 \%$ parasitaemia. Occasional pigments were evident in mature trophozoites and schizonts. Phagocytosed monocytes, macrophages and polymorphonuclear neutrophils were also detected. Different developmental stages of gametocytes - the sexual stage of the parasite, were seen in both thick and thin peripheral blood smears (Fig. 2 A to D) constituted about 
$54 \%$ of $9.5 \%$ parasitaemia. The female gametocyte or macrogametocyte is more slender and longer than the male, cytoplasm is deep blue in colour and nucleus is small, compact, staining dark red in colour, but the male gametocyte or microgametocyte is border than female, sausage shaped, cytoplasm is either pale blue or tinted pink, nucleus is staining dark pink in colour was seen in peripheral thin blood smear. Pigments and nucleus were dispersed (Fig. 2 A to D). Some fragmented gametocytes were also detected (Fig. $2 \mathrm{C}$ ). In this patient, $54 \%$ of $9.5 \%$ parasitaemia were gametocytes comprising $51 \%$ males and $49 \%$ females (1:1). Pigmented neutrophils (1.2) and monocytes $(0.4 \%)$ were present (Fig. $2 \mathrm{~A}$ and B). The unusual of marked multi parasitism asexual (14.5\%), sexual (4.5\%) comprising male gametocyte $(2.3 \%)$ and female gametocyte $(2.2 \%), 1$ parasite/RBC (4.6\%), 2 parasites/RBC $(0.4 \%), 3$ or more parasites/RBC $(0.2 \%)$, schizonts $(0.25 \%)$ and accolé/appliqué forms $1.5 \%$ were found.

A B

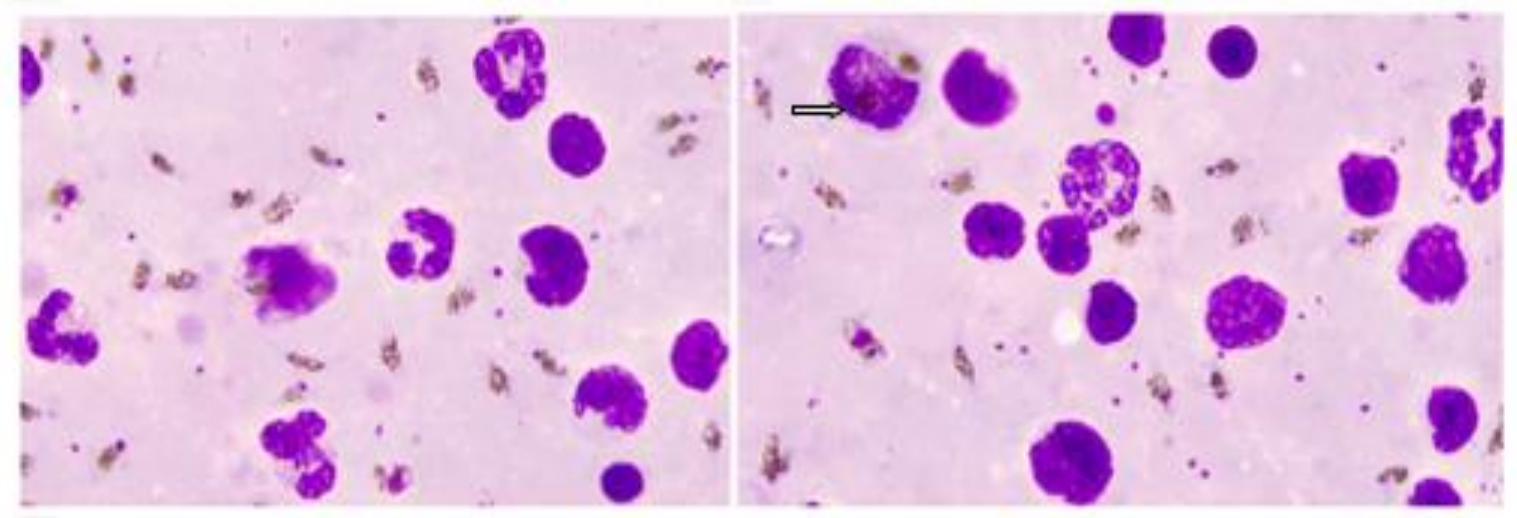

C

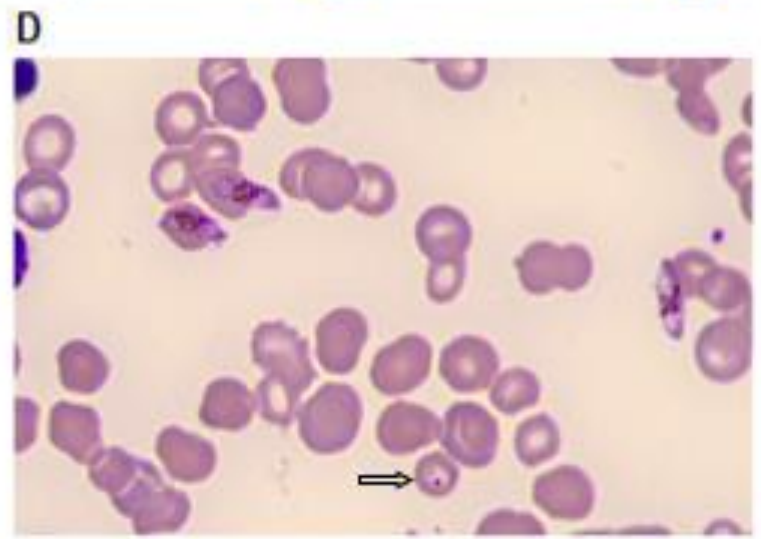

Fig. 2: Images of sexual and asexual stages of $P$. falciparum parasites (Patient 2)

Panels A and B thick smear images; arrow shows pigments of $P$. falciparum gametocyte. Plenty of gametocytes along with rings are seen on the thick smears. In $\mathrm{C}$ and $\mathrm{D}$ gametocytes on thin smears. One ring is seen at the bottom in D (arrow). X 1200. 


\section{Discussion}

Asexual hyperparasitaemia in $P$. falciparum has been observed both in children and adults by many investigators. But high gametocytaemia in $P$. falciparum is not commonly encountered. In the present study, we found 54\% gametocytaemia - to our knowledge this is the first report. Occasionally, a disastrous mistake takes place when stains and microscopes are of poor quality, microscopists misread hyperparasitaemic slides as negative [1]. Both the patients presented uncomplicated hyperparasitaemia and were treated as per national malaria drug policy and hospital protocol. Axillary temperature became normal on day 1 for both the patients. Based on their clinical assessment and prognosis, oral therapy of ACT was administered, and patients responded successfully and hyperparasitaemia started to decline. The basic definition of hyperparasitaemia needs to be updated based on transmission intensity [16]. WHO has defined hyperparasitaemia as $>5 \%$ or $250,000 / \mu \mathrm{L}$ in high transmission stable malaria areas or $>2 \%$ or $100,000 / \mu \mathrm{L}$ in low transmission areas [17]. However, in low transmission areas parasitaemia of $0.5 \%$ was considered a cutoff point for discrimination between severity levels of falciparum malaria patients [18]. Now WHO has clearly indicated that $>10 \%$ parasitaemia is considered to be severe malaria irrespective of transmission settings and patient conditions [19]. Here we calculated the parasitaemia taking the actual WBC and RBC counts, instead of WHO recommendations considering $8000 \mathrm{WBC} / \mu \mathrm{l}$ and $5 \mathrm{million} / \mathrm{mm}^{3}$ for counting and estimating parasitaemia [14]. Taking actual number gives accurate estimation especially in clinical drug trials or therapeutic efficacy studies.

The accolé/appliqué forms were seen only in hyperparasitaemia of severe $P$. falciparum malaria cases and indicate the presence of CM symptoms [20]. In the present cases, $16 \%$ and 5\% such forms were recorded, but no sign of $\mathrm{CM}$ was found, suggesting both the patients were semi-immune. The presence of $>20 \%$ mature parasite stages in peripheral blood has been associated with a poor prognosis in severe falciparum malaria $[3,20]$. However, first patient in our study who had $\geq 20 \%$ mature parasite stages still the prognosis was found normal, suggesting early diagnosis and prompt treatment are critical for a positive outcome in SM. This observation aligns with prior results where a 4-year girl child survived $70 \%$ falciparum parasitaemia in Odisha, India [21]. This patient also presented no gametocytes despite of $42 \%$ parasitaemia, which contradicts with the pervious finding, where hyperparasitaemia was associated with enhanced gametocyte carriage [4] suggesting this patient may have gametocytes neutralizing antibodies.

Mangalore city in the southwestern coast of India is endemic for malaria and contributes 70 to $80 \%$ of total malaria cases in Karnataka [22] and Anopheles stephensi is the main urban malaria vector [23]. Recent change in the malaria control operations in Mangalore [24] and national malaria control programme [23] have led to a significant reduction in malaria transmission in India. Hypergametocytaemia (54\%) with 1:1 of male and female gametocytes ratio in second patient further confirms the hypothesis that in low-transmission areas, parasites invest more in transmission to new hosts via reproduction and less in within-host replication than parasites found in high-transmission areas [25]. 


\section{Conclusion}

In summary, we show that two male adults with $P$. falciparum hyperparasitaemia were successfully recovered with the ACT and a single dose of primaquine. However, the presence of hypergametocytaemia may hinder the elimination efforts with increased infectivity, if not treated immediately. This report also signifies the need of accurately estimating the parasitaemia in malaria patients and their timely management to prevent complications and mortality.

\section{Availability of data and material}

The data used in this study are archived with Dr SK Ghosh and available from them upon reasonable request.

\section{Abbreviations:}

ACT - Artemisinin-based combination therapy

$\mathrm{SM}$ - Severe malaria

$\mathrm{CM}$ - Cerebral malaria

WBCs - White blood cells

RBCs - Red blood cells

MOD - Multi-organ dysfunction

NABL - National Accreditation Board for Testing and Calibration Laboratory

WHO - World Health Organization

HIV - Human immunodeficiency virus

HBsAg - Hepatitis B surface antigen

$\mathrm{Hb}-$ Haemoglobin

AST - Aspartate aminotransferase

ALT - Alanine transaminase 


\section{References}

1. World Health Organization. Severe malaria. Trop Med Int Health 2014; 19 (Suppl. 1): 7-131.

2. Stepniewska $\mathrm{K}$, Ashley $\mathrm{E}$, Lee $\mathrm{SJ}$, et al. In vivo parasitological measures of artemisinin susceptibility. J Infect Dis 2010; 201: 570-579.

3. Silamut K, White NJ. Relation of the stage of parasite development in the peripheral blood to prognosis in severe falciparum malaria. Trans R Soc Trop Med Hyg 1993; 87: 436-443.

4. Nacher M, Singhasivanon P, Silachamroon U, et al. Decreased hemoglobin concentrations, hyperparasitemia, and severe malaria are associated with increased Plasmodium falciparum gametocyte carriage. J Parasitol 2002; 88:97-101.

5. White NJ, Pongtavornpinyo W, Maude RJ, et al. Hyperparasitaemia and low dosing are an important source of anti-malarial drug resistance. Mal J 2009; 8:253.

6. World Health Organization. Severe and Complicated Malaria. World Health Organization, Division of Control of Tropical Diseases. Trans R Soc Trop Med Hyg 1990;84 Suppl 2:1-65.

7. Sowunmi A, Walker O, Salako LA. Hyperparasitaemia: not a reliable indicator of severity or poor prognosis in falciparum malaria in children in endemic African countries. Ann Trop Paediat 1992; 12:155-158.

8. D A Warrell, S Looareesuwan, $\mathrm{M} \mathbf{J}$ Warrell, et al. Dexamethasone proves deleterious in cerebral malaria. A double-blind trial in 100 comatose patients. N Engl J Med 1982; 306:313319.

9. Sowunmi A, Okuboyejo TM, Gbotosho GO, Happi CT. Risk factors for Plasmodium falciparum hyperparasitaemia in malarious children. BMC Infect Dis 2011; 11:268.

10. Tripathy R, Parida S, Das L, et al.Clinical manifestations and predictors of severe malaria in Indian children. Pediatr 2007; 120: e454-60.

11. Laman M, Davis TME, Manning L. Confirming cerebral malaria deaths in resource-limited settings. 2014; Am J Trop Med Hyg 90; p.192.

12. Sairam S, Domapalli S, Muthu S et al. Hematological and biochemical parameters in apparently healthy Indian opulation: defining reference intervals. Ind J Clin Biochem 2014:29(3):290-297.

13. World Health Organization. Microscopy examination of thick and thin blood films for identification of malaria parasites. Malaria microscopy standard operating procedure - MMSOP-08. Version 1. https://apps.who.int/iris/bitstream/handle/10665/274382/MM-SOP-08eng.pdf? sequence $=13 \&$ isAllowed $=y$

14. World Health Organization. Malaria parasite counting. Malaria microscopy standard operating procedure.- MM-SOP-09. Version 1. https://apps.who.int/iris/ bitstream/handle/10665/274382/ MM-SOP-09-eng.pdf?sequence $=14 \&$ is Allowed $=y$

15. National Institute of Malaria Research and National Vector Borne Disease Control Programme. Guidelines for diagnosis and treatment of malaria in India. 2014; Third Edition. New Delhi, India. pp. 1-14.

16. Wilairatana P, Tangpukdee N, Krudsood S. Definition of hyperparasitaemia in severe falciparum should be updated. Asian Pac J Trop Biomed 2013; 3: 586.

17. World Health Organization. Guidelines for the treatment of malaria. 2nd ed. Geneva: World Health Organization; 2010. p. 35.

18. Tangpukdee N, Krudsood S, Kano S, Wilairatana P. Falciparum malaria parasitemia index for predicting severe malaria. Int J Lab Hematol 2012; 34: 320-327 
19. World Health Organization. Guidelines for the treatment of malaria. 3rd ed. Geneva: World Health Organization; 2015. https://www.who.int/docs/default-source/documents/publications/ gmp/guidelines-for-the-treatment-of-malaria-eng.pdf?sfvrsn=a0138b77_2

20. Silamut K, Phu NH, Whitty C, et al. A quantitative analysis of the microvascular sequestration of malaria parasites in the human brain. Am J Path 1999; 155: 395-410.

21. Mohapatra S, Samantaray JC. Survival of a child with $70 \%$ parasitemia due to Plasmodium falciparum infection. Pediatr Infect Dis J 2012; 31:790.

22. Dayanand KK, Punnath K, Chandrashekar VN, et al. Malaria prevalence in Mangaluru city area in the southwestern coastal region of India. Mal J 2017; 15:25.

23. Ghosh SK, Rahi M. Malaria elimination in India-The way forward. J Vector Borne Dis. 2019; 56:32-40.

24. Baliga BS, Jain A, Koduvattat, N, Prakash Kumar BG, Kumar M, Kumar A, Ghosh SK. Indigenously developed digital handheld Android-based Geographic Information System (GIS)tagged tablets TABs in malaria elimination programme in Mangaluru city, Karnataka, India. Mal J 2019; 18: 444.

25. Rono MK, Nyonda M, Siman JJ, Ngoi JM, Mok S, et al. Adaptation of Plasmodium falciparum to its transmission environment. Nat Ecol Evol 2018: 2(2): 377-387.

Acknowledgements: The authors acknowledge the staff of Malaria Clinic, at the Wenlock District Government Hospital, Mangalore, Karnataka, and ICMR-National Institute of Malaria Research, New Delhi for their support to this observational study.

Funding: This study was supported from the Indian Council of Medical Research, New Delhi, India.

Authors' contributions: BM participated in fieldwork, collected clinical and epidemiological data, and laboratory analyses. AKRA and BS participated in data interpretation and critically reviewing this article. SKG carried out the microscopic examination, generated the resources, and together with HG wrote the first draft of this manuscript. All authors read, reviewed and approved the final manuscript

Ethical statement: Written prior informed consent was obtained from both the patients. The institutional review board of ICMR-National Institute of Malaria Research, New Delhi, India reviewed and approved the study (Ref. No-ECR/NIMR/EC/2012/39). The research and ethics committee of the Kasturba Medical College (KMC) under Manipal Academy of Higher Education, Mangalore, Karnataka, India, approved the study (Ref. No- IEC KMC MLR 03-16/49). All essential regulatory procedures were followed strictly.

Consent for publication: Not applicable

Competing interests: The authors declare that they have no competing interests. 


\section{Figures}

A

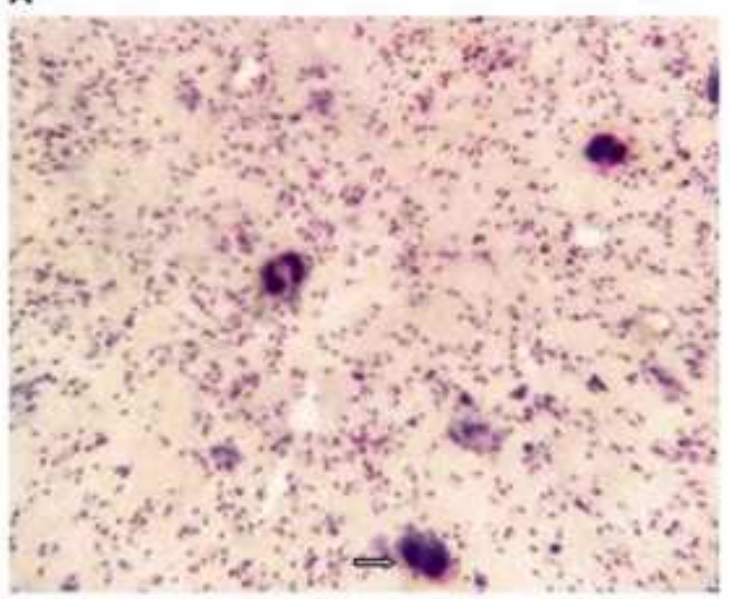

c

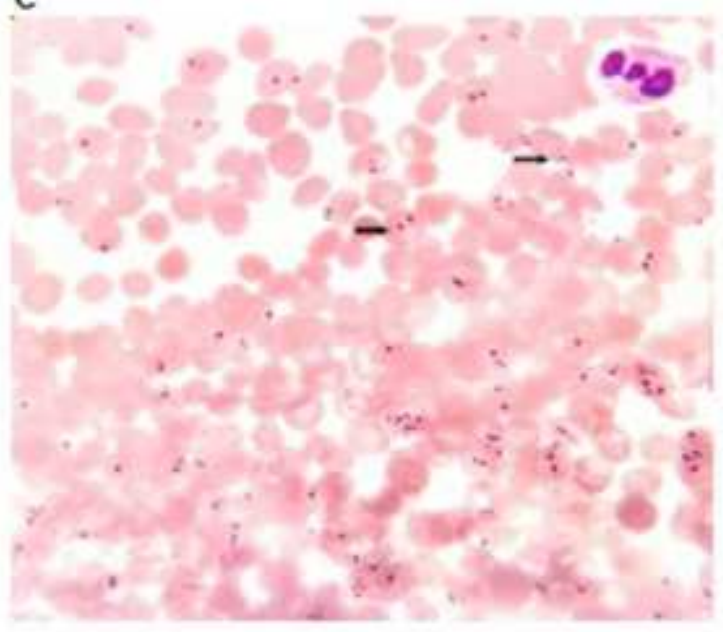

\section{B}

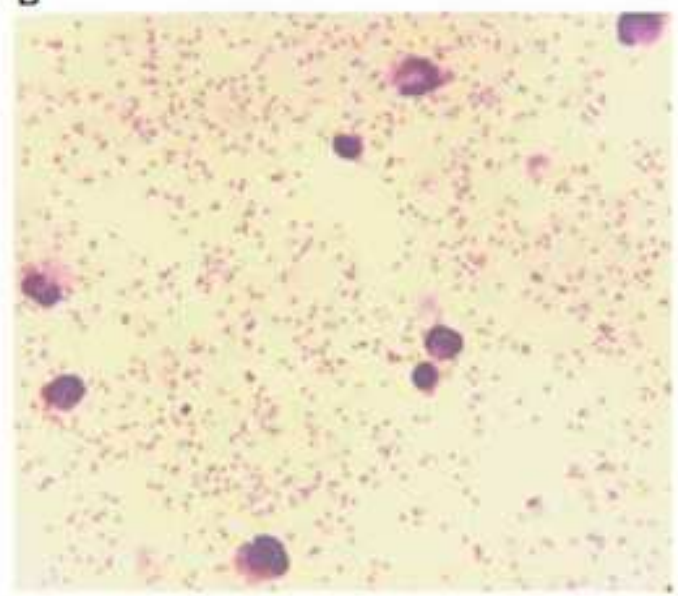

D

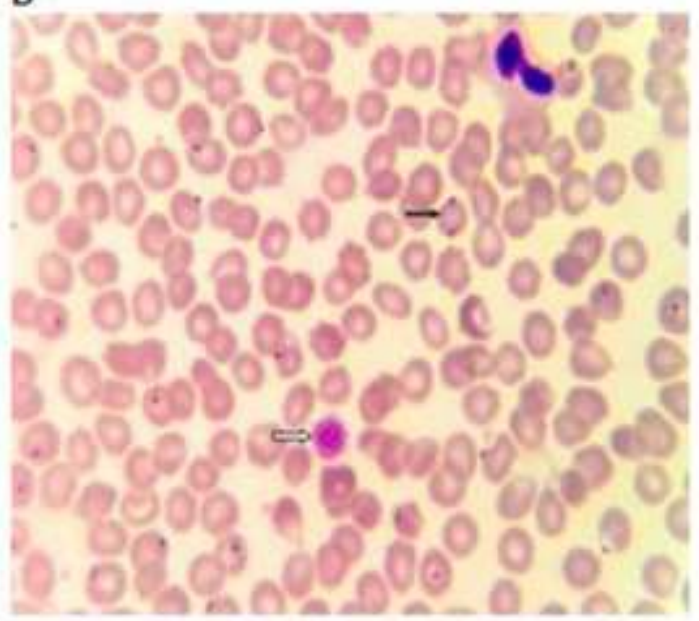

Figure 1

Images of asexual form of P. falciparum parasites X 1000 (Patient 1) Panels A and B show thick smear images with huge ring form of $P$. falciparum parasites. Panels $C$ and $D$ on thin smears. Accolé form is seen in both $\mathrm{C}$ and $\mathrm{D}$. A double-chromatin ring is seen in panel $\mathrm{C}$ (arrow in the middle). X 1000 
A B

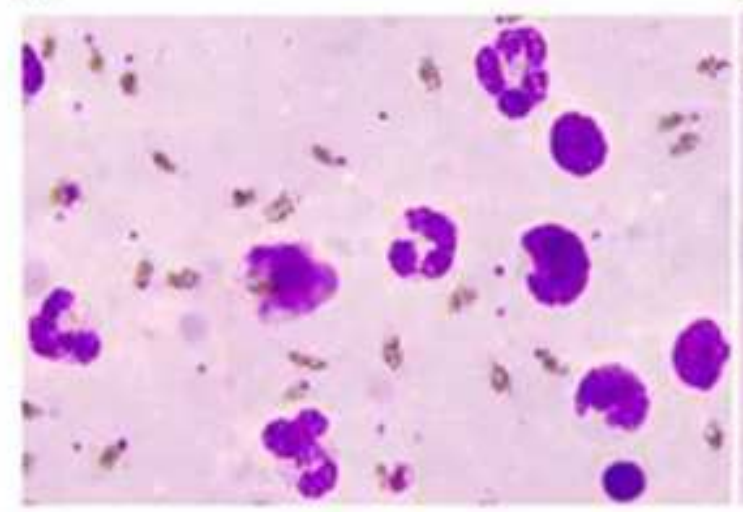

B

C
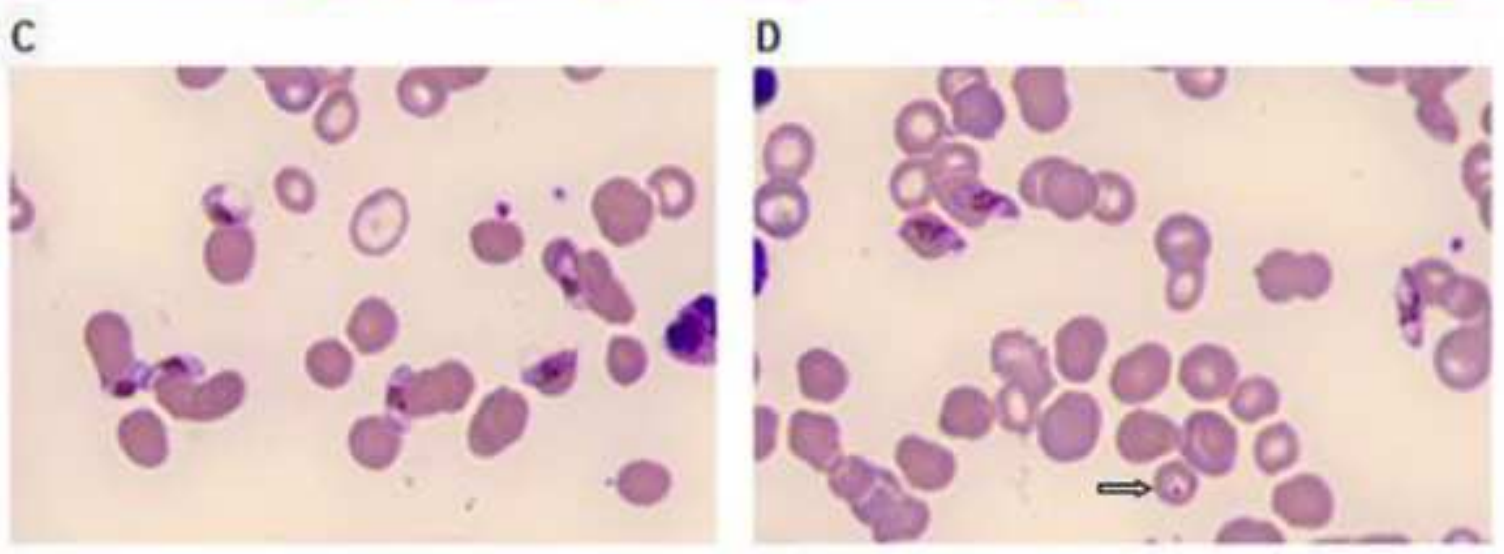

Figure 2

Images of sexual and asexual stages of P. falciparum parasites (Patient 2) Panels A and B thick smear images; arrow shows pigments of $P$. falciparum gametocyte. Plenty of gametocytes along with rings are seen on the thick smears. In $C$ and $D$ gametocytes on thin smears. One ring is seen at the bottom in $D$ (arrow). X 1200. 